\title{
DER BESONDERE FALL
}

\section{Unklare Oberbauchschmerzen \\ Diagnose „Enteritis“ lag nahe, war aber falsch}

\author{
Als bei einem 55-jährigen, bisher gesunden Patienten Oberbauchschmerzen auftraten, \\ ergab sich zunächst kein Anhalt für eine ernsthafte Erkrankung, alles sprach für eine umschriebene \\ Dünndarmentzündung. Das CT lieferte dann allerdings eine ganz andere Diagnose - \\ glücklicherweise so frühzeitig, dass der Patient noch um eine Operation herumkam.
}

Bei dem Patienten waren keine nennenswerte Vorerkrankungen bekannt. Er wurde wegen anhaltender Oberbauchschmerzen stationär eingewiesen. Die Beschwerden hatten bereits einige Tage vorher begonnen, jedoch an Intensität zugenommen. Der Stuhlgang war unauffällig, der Patient hatte kein Gewicht verloren.

\section{Klinisch unauffällig}

Bei der klinischen Untersuchung erschien das Abdomen leicht gebläht. Bei der Palpation gab der Patient einen leichten, diffusen Druckschmerz an, es lagen weder Resistenz noch Abwehrspannung vor, die Darmgeräusche waren normal. Auch die Laborparameter inkl. Entzündungswerte, Lipase und Amylase lagen im Normbereich. Sonografisch erschien ein Dünndarmsegment etwas verdickt, sodass zunächst eine Enteritis diskutiert und eine Untersuchung des Stuhls auf pathogene Keime veranlasst wurde. Zum Ausschluss einer gastralen Ursache wurde auch eine Ösophago-GastroDuodenoskopie durchgeführt, die ebenfalls keinen krankhaften Befund ergab.

\section{CT führt zur Diagnose}

Als am folgenden Tag die Beschwerden weiter zunahmen, wurde die Oberbauchsonografie wiederholt. Auch jetzt stellte sich eine obere Jejunalschlinge verdickt dar. Daraufhin wurde ein CT mit Angio durchgeführt. Hier zeigte sich ein thrombotischer Verschluss der Vena mesenterica superior mit Thromboseausläufer in die Pfortader. Die arterielle Perfusion war nicht beeinträchtigt.

Daraufhin wurde eine Antikoagulation mit einem niedermolekularen $\mathrm{He}$ -

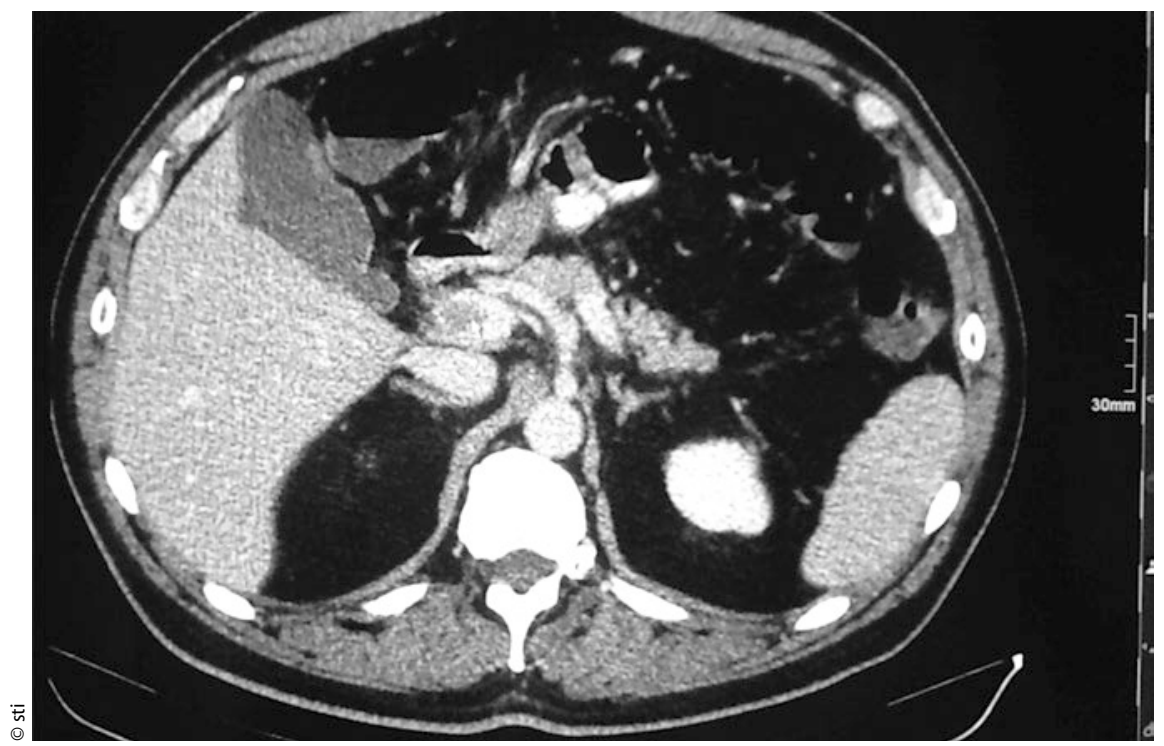

Computertomogramm: thrombotisches Material in der Pfortader. parin eingeleitet. Im weiteren Verlauf wurde der Patient rasch vollkommen beschwerdefrei. Angesichts der ungewöhnlichen Manifestation der Thrombose wurde ein Thrombophiliescreening veranlasst. Dieses ergab eine hereditäre Thrombophilie, und zwar eine heterozygote APC-Resistenz (Faktor-V-Leyden), sodass eine dauerhafte orale Antikoagulation indiziert war.

\section{Unspezifische Symptomatik}

Die Mesenterialvenenthrombose ist in ca. 20\% der Fälle die Ursache einer mesenterialen Ischämie, also sehr viel seltener als ein arterieller Verschluss. Die Symptomatik ist unspezifisch mit Übelkeit, Erbrechen und Bauchschmerzen. Ursachen können sein: Traumen, Z.n. Whipple-Operation, Pankreatitis und Thrombophilie wie APC-Resistenz oder Antiphospholipid-Antikörper-Syndrom. In den meisten Fällen ist eine Darmresektion erforderlich, in seltenen Fällen ist eine Thrombektomie oder eine Antikoagulation ausreichend. Die Prognose ist deutlich besser als bei einem Mesenterialarterienverschluss. Es besteht immer die Indikation für eine Langzeitantikoagulation.

Dr. Peter Stiefelhagen

\section{FABULA DOCET}

Bei plötzlich einsetzenden abdominellen Schmerzen muss nach Ausschluss häufigerer Ursachen auch an eine vaskuläre Erkrankung gedacht und eine solche mittels CT ausgeschlossen werden. Liegt eine Mesenterialvenenthrombose vor, so ist immer ein Thrombophiliescreening indiziert. 